\title{
Coherence expansion and polariton condensate formation in a semiconductor microcavity
}

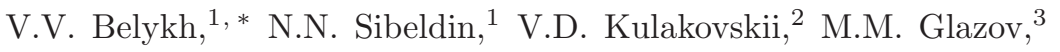 \\ M.A. Semina, ${ }^{3}$ C. Schneider, ${ }^{4}$ S. Höfling, ${ }^{4}$ M. Kamp,${ }^{4}$ and A. Forchel ${ }^{4}$ \\ ${ }^{1}$ P.N. Lebedev Physical Institute, Russian Academy of Sciences, Moscow, 119991 Russia \\ ${ }^{2}$ Institute of Solid State Physics, Russian Academy of Sciences, Chernogolovka, 142432 Russia \\ ${ }^{3}$ Ioffe Physical-Technical Institute of the Russian Academy of Sciences, St. Petersburg, 194021 Russia \\ ${ }^{4}$ Technische Physik, Physikalisches Institut and Wilhelm Conrad Röntgen Research Center for Complex Material Systems, \\ Universität Würzburg, D-97074 Würzburg, Germany
}

\begin{abstract}
The dynamics of the expansion of the first order spatial coherence $g^{(1)}$ for a polariton system in a high-Q GaAs microcavity was investigated on the basis of Young's double slit experiment under 3 ps pulse excitation at the conditions of polariton Bose-Einstein condensation. It was found that in the process of condensate formation the coherence expands with a constant velocity of about $10^{8} \mathrm{~cm} / \mathrm{s}$. The measured coherence is smaller than that in thermally equilibrium system during the growth of condensate density and well exceeds it at the end of condensate decay. The onset of spatial coherence is governed by polariton relaxation while condensate amplitude and phase fluctuations are not suppressed.
\end{abstract}

PACS numbers: 78.67.Pt, 71.36.+c, 78.47.jd, 03.75.Kk

One of the most important characteristics of BoseEinstein condensate is the spatial coherence or the offdiagonal long range order, i.e. the property of the system to share the same wave function at different points separated by a distance larger than the thermal de Broglie wavelength. To understand the processes governing the Bose-Einstein condensation (BEC), it is important to know how fast the coherence is established throughout the system during the condensate formation. This question was addressed theoretically in Refs. [1, 2], where it was shown that in the process of a condensation particles first relax to the low energy or so-called coherent region where the kinetic energy of the particle is of the order of its interaction energy with other particles. Second, the fluctuations of density are smoothed out and a "quasicondensate" is formed. Further, the phase fluctuations disappear resulting in the long range order formation within the system signifying the onset of the "true condensate". The timescales of these processes are quite different: the relaxation to the low energy region is mainly determined by the stimulated scattering processes, whereas the quasicondensate formation is governed by the interparticle interactions. Experimentally, the dynamics of spatial coherence formation was first studied for a gas of ultracold atoms in Ref. [3], where it was found that the coherence expands with a constant velocity of about $0.1 \mathrm{~mm} / \mathrm{s}$.

In this Letter we discuss the expansion of the spatial coherence in a condensate of mixed exciton-photon states, polaritons, in a semiconductor microcavity (MC) with embedded quantum wells. The bosonic statistics of these particles and the extremely light effective mass $m$ $\left(\sim 10^{-4}\right.$ of the free electron mass $\left.m_{e}\right)$ allow observing MC polariton BEC up to the room temperatures, which inspired a considerable attention to this system in the last decade. Up to now a number of bright phenomena in the
MC polariton system have been observed and discussed: polariton BEC [4], superfluidity [5], quantized vortices 6], spin-Meissner 4] and Josephson effect [8] (see [9] for a review). Compared with atomic BEC, the MC polariton condensation is highly specific: the polariton system is strongly nonequilibrium due to the short lifetime [10].

The first order spatial coherence function characterizes the ability of the polariton system to interfere [11], and is related to off-diagonal elements of density matrix $\varrho\left(\boldsymbol{\rho}_{1}, \boldsymbol{\rho}_{2}\right)$ in the coordinate space:

$$
g^{(1)}\left(\boldsymbol{\rho}_{1}-\boldsymbol{\rho}_{2}\right)=\frac{\varrho\left(\boldsymbol{\rho}_{1}, \boldsymbol{\rho}_{2}\right)}{\sqrt{\varrho\left(\boldsymbol{\rho}_{1}, \boldsymbol{\rho}_{1}\right) \varrho\left(\boldsymbol{\rho}_{2}, \boldsymbol{\rho}_{2}\right)}} .
$$

The $g^{(1)}$ can be probed in the MC polariton system by measuring the interference of the light emitted from different points on the sample, since the amplitude and phase of the electric field of the cavity emission is directly proportional to the amplitude and phase of the wavefunction of the polariton condensate [4, 12, 13]. Dynamics of $g^{(1)}$ in a process of MC polariton condensation was studied experimentally for the first time in a CdTebased $\mathrm{MC}$ for the fixed separation between condensate regions [14] and very recently in a GaAs-based MC [15]. In the present work the spatial coherence dynamics of the polariton system in a high-Q GaAs-based MC is studied in detail for different separations $\Delta x$ between condensate regions. As a result it was found for the first time that the coherence expands with almost constant velocity and its value was measured. By tracing the dependencies $g^{(1)}(\Delta x)$ at different times we extract the dynamics of the coherence length $r_{c}$. We have found that in the time range of the condensate decay, the coherence is larger than that in the equilibrium system, which is highly unexpected. Our study indicates that in a polariton system under pulsed excitation of the MC the formation of the 
spatial coherence is governed by the processes of polariton relaxation towards the ground state, whereas amplitude and phase fluctuations of the quasicondensate are not suppressed.

The sample is a half wavelength MC with Bragg reflectors made of 32 (for the top mirror) and 36 (for the bottom mirror) $\mathrm{AlAs}$ and $\mathrm{Al}_{0.13} \mathrm{Ga}_{0.87} \mathrm{As}$ pairs. It has a Q-factor of about 7000 and the Rabi splitting of 5 $\mathrm{meV}$. The experiments were performed at $T=10 \mathrm{~K}$ and photon-exciton detuning of $-9 \mathrm{meV}$. The sample was excited by the radiation of a mode-locked Ti-sapphire laser generating a periodic $(f=76 \mathrm{MHz})$ train of 2.5-ps-long pulses at the reflection minimum of the mirror $11 \mathrm{meV}$ above the bare exciton energy. The beam was focused in a $20 \mu \mathrm{m}$ spot on the sample surface. The spot was imaged with magnification of $\Gamma=6$ on the light-absorbing plate with two transparent parallel slits [12]. The interference pattern of the emission coming from the regions of the sample selected by the two slits was formed on the slit of the Hamamatsu streak camera, operating with time resolution of 3 ps. Spatial coherence $g^{(1)}$ was extracted as the visibility of the interference pattern $g^{(1)}=\left(I_{\max }-I_{\min }\right) /\left(I_{\max }+I_{\min }\right)$, where $I_{\min }$ and $I_{\max }$ are minimal and maximal intensities within one period of interference pattern, averaged over all the observed periods. The time-resolved MC emission spectra were recorded by a spectrometer coupled to the streak camera with spectral (temporal) resolution of $0.25 \mathrm{meV}(20 \mathrm{ps})$.

To convert the intensity $I(t)$, measured by the streakcamera to the number of polaritons $N$ at states with wavevectors $|k|<3 \mu \mathrm{m}^{-1}$ (the collection aperture is $40^{0}$ ), the integrated intensity of the MC photoluminescence (PL) $I_{P L}$ was measured by the sensitive power meter. The number of polaritons was evaluated by the relation $N(t)=2 I_{P L} \tau_{L P} I(t) /\left(f \hbar \omega \int I(t) d t\right)$, where factor 2 takes into account two directions of photon emission, $\hbar \omega$ is the energy of emitted photons, $\tau_{L P} \approx 3$ ps is the polariton lifetime at the bottom of the lower polariton (LP) branch.

At low excitation density $P$ the PL dynamics of the LP branch is relatively slow, and the angular distribution of intensity indicates a bottleneck effect. As $P$ is increased above the BEC threshold $P_{t h r}=0.7 \mathrm{~kW} / \mathrm{cm}^{2}$ (this value corresponds to the time-averaged power of pulsed excitation), a fast and intense component in the PL dynamics corresponding to $k \approx 0$ appears. Onset of the fast component is accompanied by the blueshift of the spectral line and decrease of its width (Fig. 11). The energy position of the spectral line is close the bare MC mode $E_{M C}$ just after the excitation pulse and relaxes to the energy of LP mode $E_{L P}$ with time. The maximum population (inset in Fig. 1) is reached when the spectral line energy is between the MC and LP modes indicating that BEC is observed in the strong coupling regime despite the high particle density [16 18].

Below $P_{t h r}$ interference fringes in the double slit ex-

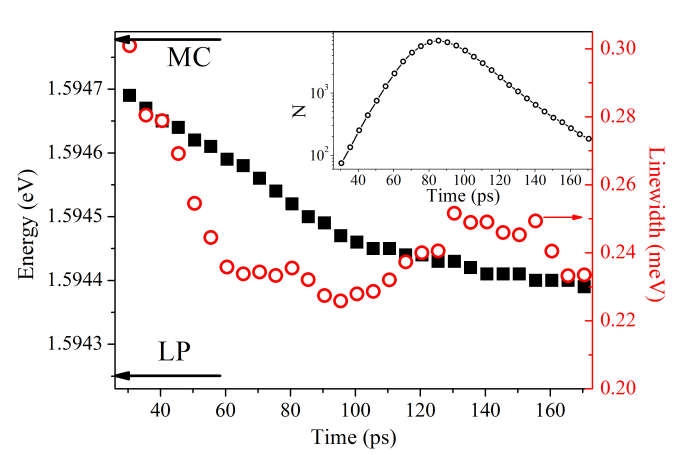

FIG. 1: Dynamics of the energy (full squares, left axis) and FWHM (empty circles, right axis) of the LP emission line. $E_{M C}$ and $E_{L P}$ are marked by black arrows. Inset shows the number of LPs near $k=0$. Excitation power is $1.8 P_{t h r}$.

periment are observed only for the smallest studied slits separation, and $g^{(1)}(\Delta x=3 \mu \mathrm{m})<0.3$ at $0.9 P_{t h r}$. Above $P_{t h r}$ the interference fringes are well resolved up to $\Delta x=$ $20 \mu \mathrm{m}$ (Fig. 2(a)). The dynamics of $g^{(1)}$ for different $\Delta x$ at $P=1.8 P_{t h r}$ is presented in Fig. 2(b) together with the dynamics of the polariton number $N$ at the bottom of the LP branch. Figure 2(b) shows that the maximum value of $g^{(1)}$ decreases with increased $\Delta x$ and the decay of $g^{(1)}$ in the whole range of $\Delta x$ occurs much slower than that of the condensate density in agreement with [14].

Interestingly, Fig. 2(b) shows that the coherence buildup time increases with an increase of $\Delta x$, indicating a finite velocity of the coherence expansion. We define the coherence buildup time $t_{0.5}(\Delta x)$ as the time when $g^{(1)}$ reaches half of its maximum value for a given $\Delta x$. These times are marked by arrows in Fig. 2(b). Figure 2(c) shows that the dependence of $t_{0.5}$ on $\Delta x$ is close to linear indicating that the coherence expands with almost constant velocity $v_{c}=0.6 \cdot 10^{8} \mathrm{~cm} / \mathrm{s}$.

Figure 3 shows the dynamics of the coherence length $r_{c}$, which is defined from $g^{(1)}\left(r_{c}\right)=1 / e$, and number of polaritons at the bottom of the LP branch $N$ for $P>P_{t h r}$. At the beginning of the BEC, $r_{c}$ grows almost linearly with time, reaches its maximum and decays afterwards. It follows from Fig. 3 that $r_{c}$ and $N$ reach their maximal values almost at the same time, but the decay of $N$ at high $P$ occurs much faster than that of $r_{c}: N$ decays with the lifetime of $20-30 \mathrm{ps}$ whereas $r_{c}$ decreases at most by $50 \%$ during the first $30-40$ ps and then remains nearly constant during the next several tens of picoseconds where $N$ decreases by more than an order of magnitude. Furthermore, Fig. 3 shows that the buildup of the coherence at $P=1.2 P_{t h r}$ begins when the particle number $N$ is more than one order of magnitude smaller than that at $P=4.2 P_{t h r}$ and that the maximal value of $r_{c}$ decreases with $P$ at $P>2 P_{t h r}$ in spite of a strong (about an two orders of magnitude) increase of 

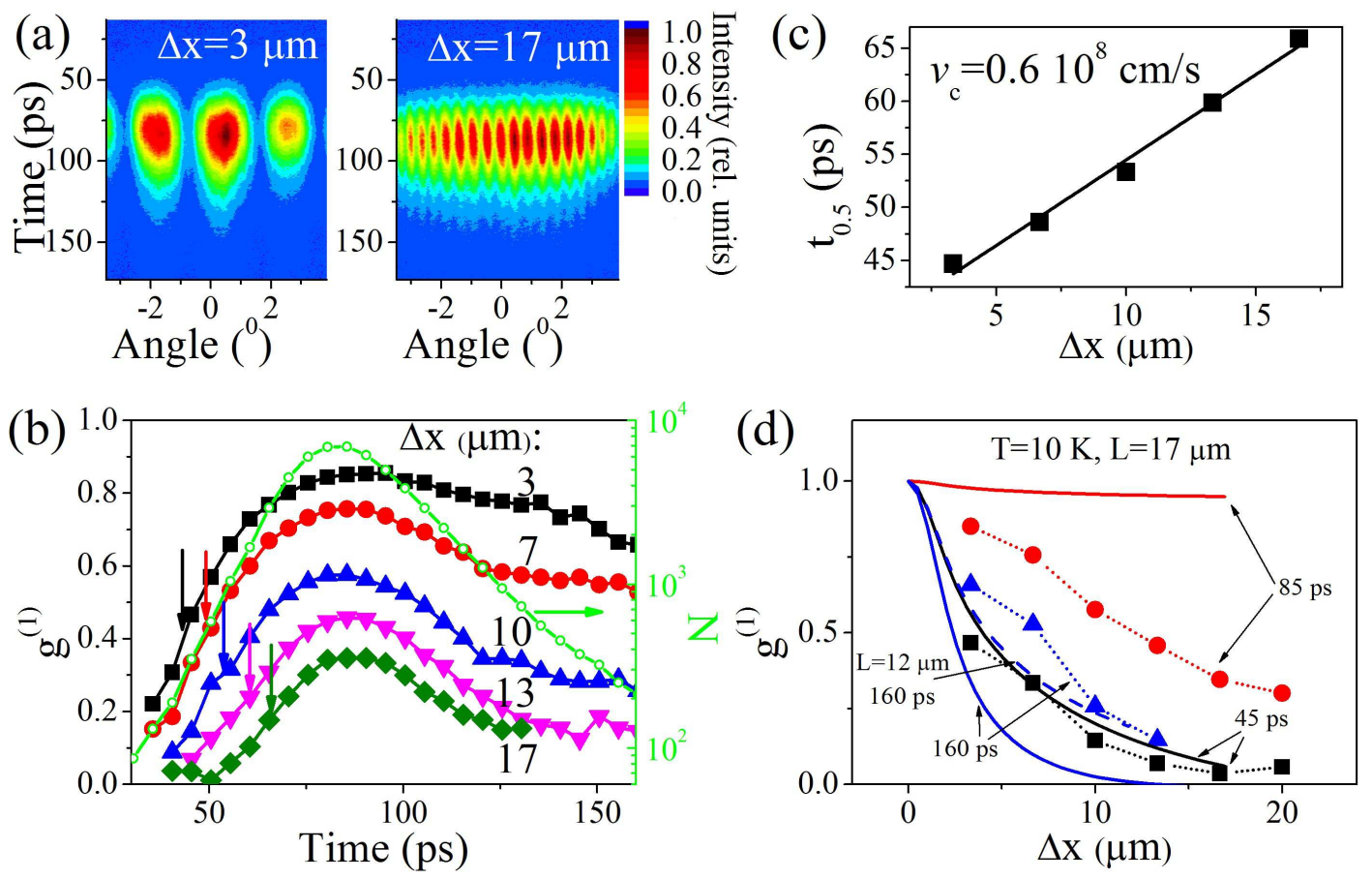

FIG. 2: (a) Streak camera images of the interference patterns of the emission coming from regions of the sample separated by $\Delta x=3$ and $17 \mu \mathrm{m}$. Horizontal axis corresponds to the angle of the emission passed through the slits with respect to the sample normal. (b) Dynamics of the spatial coherence for different $\Delta x$ (left axis) and number of particles near the LP branch bottom (right axis). Arrows mark the times $t_{0.5}$, when $g^{(1)}$ reaches half of its maximal value. (c) Dependence of the coherence buildup time $t_{0.5}$ on $\Delta x$. Solid curve shows linear fit. (d) Dependences $g^{(1)}(\Delta x)$ at different times $t$. Experimental results are shown by symbols, solid lines show dependences calculated at the same times as experimental ones for the condensate size $L=17 \mu \mathrm{m}$, and blue dashed line is dependence calculated at $t=160 \mathrm{ps}$ and $L=12 \mu \mathrm{m}$. Excitation power for (a)-(d) is $1.8 P_{t h r}$.

the condensate density. These facts show that the length of coherence, as well as the rate of its increase, is not solely defined by the condensate occupation because of the nonequilibrium nature of the polariton BEC.

In a $2 \mathrm{D}$ system of a finite size $L$ under the conditions of thermal equilibrium the number of particles in the ground state can be estimated as

$$
N_{0}=N-N^{\prime}=N-\int_{1 / L}^{\infty} \frac{k d k L^{2}}{\pi}\left(e^{\frac{\hbar^{2} k^{2}}{2 m k_{B} T}}-1\right)^{-1},
$$

where $N$ is the total number of LPs, $N^{\prime}$ is the number of LPs in all states but the ground, $k_{B}$ is the Boltzmann constant. Here the chemical potential $\mu=0$.

It follows from Figs. 2(b) and 3 that the onset of spatial coherence at $P<2 P_{t h r}$ starts at $N \sim 10^{2}$. This value is less than the estimated from Eq. (2) $N^{\prime} \approx 400$ at $T=10 \mathrm{~K}$ in the investigated LP system with the lateral size $L \approx 17 \mu \mathrm{m}$ and $m=5 \cdot 10^{-5} m_{e}$. Thus, the formation of spatial coherence starts at negative chemical potential $\mu$ with respect to the bottom of the LP branch (e.g. $\mu \sim-0.05 \mathrm{meV}$ for $P=1.8 P_{t h r}, t=40 \mathrm{ps}$ ) and hence governed by the relaxation process of polaritons to the low energy region, while interaction-induced suppression of amplitude and phase fluctuations plays no role [1, 2]. We note also, that at $P<2 P_{t h r}$ the estimated value of the interaction energy for the condensed polaritons $\Delta E=\alpha N_{0} / L^{2}<2 \mu \mathrm{eV} \ll k_{B} T \approx 1 \mathrm{meV}$ (where $\alpha=10^{-12} \mathrm{meVcm}^{2}$ is the polariton-polariton interaction constant). Hence, $g^{(1)}$ should be close to that for the classical noninteracting gas with the particle distribution function $N_{k}$ (see Eq. (11) and [12]):

$g^{(1)}(\Delta x)=\frac{\sum_{k} N_{k} \mathrm{e}^{\mathrm{i} k \Delta x}}{\sum_{\boldsymbol{k}} N_{k}}=\frac{\frac{L^{2}}{\pi} \int_{1 / L}^{\infty} J_{0}(k \Delta x) N_{k} k d k+N_{0}}{\frac{L^{2}}{\pi} \int_{1 / L}^{\infty} N_{k} k d k+N_{0}}$,

where $J_{0}(x)$ is the zero order Bessel function.

The detailed modeling of the polariton distribution similar to that in Refs. [19] is beyond the scope of the present paper. Here we discuss the reason of the difference between the experimental dependences $g^{(1)}(\Delta x)$ and calculated ones for the thermal Bose distribution of LPs with the use of $T=10 \mathrm{~K}$ and $\mu$ determined from the measured polariton number $N$ in the low energy region. The difference between the calculated and measured curves indicates how far the system is from the thermal equilibrium.

The results of the calculation for three consecutive stages: condensation onset, maximum condensate den- 
sity and its decay, are shown in Fig. 2(d) for $P=1.8 P_{t h r}$ and the condensate size $L=17 \mu \mathrm{m}$ determined from the experiment. It is seen that the experimental dependence $g^{(1)}(\Delta x)$ is slightly below the calculated thermally equilibrium one in the first stage $(t=45 \mathrm{ps})$, the difference strongly increases in the second stage $(t=85 \mathrm{ps})$ whereas in the third stage $(t=160 \mathrm{ps})$ experimental values $g^{(1)}(\Delta x)$ turn out well above the calculated ones. This result is especially surprising as it indicates that the occupation of $k \approx 0$ states with respect to that of higher-energy states exceeds the thermally equilibrium one, i.e. the effective polariton temperature is lower than the bath temperature in the time range of condensate decay. Measurements of polariton distribution along the LP branch (not shown) indicate that the distribution function approaches the thermal one at the onset of condensation, but the occupation numbers of low $k \neq 0$ states are slightly increased compared to the thermal values, which explains a small discrepancy between the experimental and calculated $g^{(1)}(\Delta x)$ at $t=45 \mathrm{ps,}$ when $N \approx 350<N^{\prime}$.

An increase in the discrepancy between the experimental and calculated $g^{(1)}(\Delta x)$ in the range of maximal $N$ at $t=85$ ps when $N \approx 7000 \gg N^{\prime}$ indicates that the LP system becomes more nonequilibrium at high condensate density. The most probable reason for that is the runaway of condensed polaritons from the small bounded $(\sim 20 \mu \mathrm{m})$ photoexcited region due to their repulsive interaction with a dense exciton reservoir 20]. In addition, for the equilibrium system with $N_{0} \gg N^{\prime}$, the coherence is defined by the amplitude and phase fluctuations of the ground state wavefunction not taken into account in Eq. (3). Indeed, the quasicondensate amplitude fluctuations are suppressed during the time $\tau_{A} \approx \hbar L^{2} /(\alpha N)$ [1, 2], determined by the interparticle interaction. For $N=7000, L=17 \mu \mathrm{m}$ we obtain value $\tau_{A} \sim 0.3 \mathrm{~ns}$ that exceeds the lifetime of LP condensate at $P=1.8 P_{t h r}$ (Fig. (3)). It follows then, that the amplitude and, especially, phase fluctuations are not suppressed.

Finally, let us discuss the reason for the unexpectedly high coherence in the decaying polariton system at $t \sim 160$ ps with $N \approx 200<N^{\prime}$ which exceeds markedly the coherence in the thermally equilibrium system. Note that the calculations underestimate experimental values of $g^{(1)}$ even for the unrealistically small condensate size $L=12 \mu \mathrm{m}$ (dashed line in Fig. 22d). At large $t>100 \mathrm{ps}$ the reservoir becomes highly depleted, and the equilibrium in the system is established mainly via ineffective excitation of condensed polaritons by acoustic phonons with characteristic time exceeding the condensate decay time $\tau$. In this case the wave function at a given distance $\boldsymbol{\rho}$ decays as $\psi(\boldsymbol{\rho}) \propto \exp \left(-\frac{t}{2 \tau}\right)$. Thus, both diagonal and off-diagonal elements of the polariton density matrix decay approximately with the same rate $1 / \tau$ resulting in a nearly constant ratio of the LP numbers in the ground state to that in the excited states well exceeding the equi-

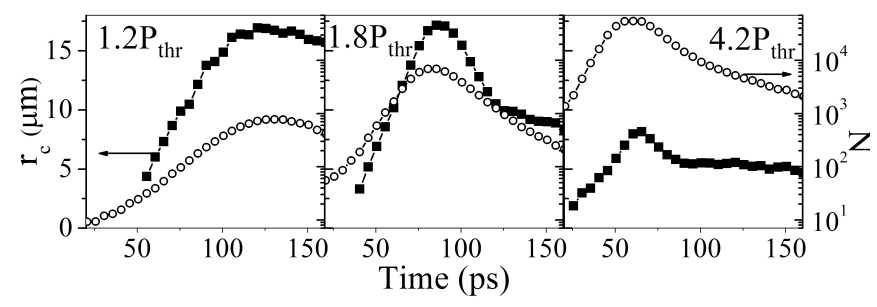

FIG. 3: Dynamics of the coherence length (full squares, left axes, linear scales) and number of particles near the bottom of the LP branch (empty circles, right axes, logarithmic scales) at different excitation powers.

librium ratio at long times. As a result, $g^{(1)}$ weakly decays with time in agreement with the experiment.

It is worth to compare the measured coherence expansion velocity $v_{c}$ for the polariton condensate (about $10^{8} \mathrm{~cm} / \mathrm{s}$ ) and that for the atomic condensate (about $\left.10^{-2} \mathrm{~cm} / \mathrm{s}[3]\right)$. The buildup of the spatial coherence is determined by the relaxation of the particles to the ground state and manifests itself by the condition $r_{c}>$ $\lambda_{d B}$, where $\lambda_{d B}$ is thermal de Broglie wavelength. So one can estimate the coherence expansion velocity as $v_{c} \sim \lambda_{d B} / \tau_{\text {rel }}$. For polaritons $\lambda_{d B} \sim 2 \mu \mathrm{m}$ at $T=10 \mathrm{~K}$ and for atoms $\sim 0.4 \mu \mathrm{m}$ at $T=0.2 \mu \mathrm{K}$, so the large difference in $v_{c}$ is related to the difference in the relaxation times $\tau_{\text {rel }}$, which is $\sim 10 \mathrm{ps}$ for polaritons and $\sim 100 \mathrm{~ms}$ for atoms [3]. The relaxation for both polaritons and atoms is accomplished via interparticle scattering, while for polaritons scattering with phonons also plays role. The rate of the interparticle collisions depends on the scattering cross sections and on the average velocity of the particles. The latter is determined by the particle mass and temperature which differ in many orders of magnitude ensuring the necessary ratio of the relaxation times for polaritons and atoms.

To conclude, we have studied the dynamics of the spatial coherence for a LP condensate under pulsed ps-long excitation for different excitation powers. It has been found that in the process of condensate formation, first order coherence expands with almost constant velocity of about $10^{8} \mathrm{~cm} / \mathrm{s}$. We have shown that the coherence is influenced by polariton relaxation from the reservoir. The onset of spatial coherence is determined by the narrowing of polariton distribution in $k$-space rather than formation of the condensate phase, and at high excitation density coherence is limited by condensate amplitude fluctuations. The true condensate i.e. macroscopic occupation of the ground state with suppressed phase fluctuation is not achieved under ps-long pulsed pumping.

We are grateful to D.A. Mylnikov for help in the experiment and S.S. Gavrilov, N.N. Gippius, L.V. Keldysh, A.V. Sekretenko, S.G. Tikhodeev and V.B. Timofeev for valuable advice and useful discussions. This study was supported by the RFBR (projects no. 11-02-01310, 11- 
02-12261, 11-02-00573), RAS, Ministry of Education and Science of the Russian Federation (contract no. 8680), the State of Bavaria, and EU projects POLAPHEN and SPANGL4Q. MAS was partially supported by the RF President Grant NSh-2901.2012.2.

* Electronic address: belykh@lebedev.ru

[1] Yu.M. Kagan, B.V. Svistunov, and G.V. Shlyapnikov, JETP 75, 387 (1992).

[2] Yu. Kagan and B.V. Svistunov, JETP 78, 187 (1994).

[3] S. Ritter et al., Phys. Rev. Lett. 98, 090402 (2007).

[4] J. Kasprzak et al., Nature 443, 409 (2006).

[5] A. Amo et al., Nature Phys. 5, 805 (2009).

[6] K.G. Lagoudakis et al., Nature Phys. 4, 706 (2008).

[7] A.V. Larionov et al., Phys. Rev. Lett. 105, 256401 (2010).

[8] K.G. Lagoudakis, B. Pietka, M. Wouters, R. Andre, and B. Deveaud-Pledran, Phys. Rev. Lett. 105, 120403 (2010).

[9] D. Sanvitto and V. Timofeev (editors), Exciton Po- laritons in Microcavities (Springer, New York, 2012), Springer Series in solid-state sciences Vol. 172.

[10] F. Tassone, C. Piermarocchi, V. Savona, A. Quattropani, and P. Schwendimann, Phys. Rev. B 56, 7554 (1997).

[11] O. Penrose, L. Onsager, Phys. Rev. 104, 576 (1956); Roy J. Glauber, Phys. Rev. 131, 2766 (1963).

[12] H. Deng, G.S. Solomon, R. Hey, K.H. Ploog, Y. Yamamoto, Phys. Rev. Lett. 99, 126403 (2007).

[13] G. Roumpos et al., PNAS 109, 6467 (2012).

[14] G. Nardin et al., Phys. Rev. Lett. 103, 256402 (2009).

[15] H. Ohadi et al., Phys. Rev. Lett. 109, 016404 (2012).

[16] J. Keeling, P.R. Eastham, M.H. Szymanska, P.B. Littlewood, Phys. Rev. B 72, 115320 (2005).

[17] K. Kamide and T. Ogawa, Phys. Rev. Lett. 105, 056401 (2010).

[18] T. Byrnes, T. Horikiri, N. Ishida, Y. Yamamoto, Phys. Rev. Lett. 105, 186402 (2010).

[19] G. Malpuech et al., Semicond. Sci. Technol. 18, S395 (2003); D. Sarchi and V. Savona, Phys. Rev. B 75, 115326 (2007); T.D. Doan, H.T. Cao, D.B.Tran Thoai, H. Haug, Phys. Rev. B 78, 205306 (2008).

[20] A.S. Brichkin et al., Phys. Rev. B 84, 195301 (2011). 